\title{
Improving Knowledge Structure through Extended Scratch-Build Concept Mapping
}

\author{
Didik Dwi Prasetya ${ }^{a, b, 1, *}$, Yusuke Hayashi ${ }^{a, 2}$, and Tsukasa Hirashima ${ }^{a, 3}$ \\ ${ }^{a}$ Department of Information Engineering, Hiroshima University, Japan \\ ${ }^{\mathrm{b}}$ Department of Electrical Engineering, Universitas Negeri Malang, Malang, Indonesia \\ ${ }^{1}$ didikdwi@um.ac.id, ${ }^{2}$ hayashi@ @lel.hiroshima-u.ac.jp, ${ }^{3}$ tsukasa@lel.hiroshima-u.ac.jp
}

\begin{tabular}{|c|c|}
\hline Article Info & ABSTRACT \\
\hline $\begin{array}{l}\text { Keyword: } \\
\text { Concept map } \\
\text { Knowledge structure } \\
\text { Open-Ended } \\
\text { Extended Scratch-Build }\end{array}$ & $\begin{array}{l}\text { Extended concept mapping is a potential technique to elicit missing ideas and } \\
\text { relationships. This study explored an Extended Scratch-Build (ESB) concept mapping } \\
\text { in improving learners' knowledge structure. ESB is an expansion of an open-ended } \\
\text { approach that asks students to connect the prior-existing original concept map with the } \\
\text { new additional map on related material topics. The practical use of ESB has been } \\
\text { conducted and proves positive effects on learning achievements. However, no } \\
\text { information has been provided regarding the performance of the concept map } \\
\text { components that describe the broad overview of students attainments. This } \\
\text { observational study focused on improving learners knowledge using quantity } \\
\text { measurements based on concept map features, consists of a number of concepts, } \\
\text { number of links, and a number of propositions. University students }(\mathrm{N}=27) \text { with a } \\
\text { major in Informatics Engineering participated in the study. The results reported that } \\
\text { students' knowledge structure on additional maps significantly increased compared to } \\
\text { the prior original map. }\end{array}$ \\
\hline
\end{tabular}

\section{INTRODUCTION}

Concept maps are graphical tools that are recognized for representing the conceptual knowledge structure of individuals accurately. Concept maps can be used for knowledge construction, teaching, learning, and assessment. Various fields and education levels have implemented concept maps and perceive the benefits of learning engagement and enhancement. The primary purpose of concept mapping is a knowledge representation in the visual format in order to acquire an overview of a domain of knowledge. Individual ideas are described as nodes that are connected to other nodes with linking words that represent propositions. A proposition represents a unit's declarative or component of knowledge that presents particular meaning.

Concept maps construction style is classified into two types: (1) closed-ended; and (2) open-ended [1]. The closedended or high-directed concept mapping style provides finite concepts, connecting lines, and linking phrases regarding the teacher's map. This approach facilitates automatic assessment by comparing students' and teacher's maps [2]. In contrast, the open-ended or low-directed style allows learners to use any concepts and any linking words related to the particular topic in their diagrams. This condition enables the teacher to uncover different knowledge structures across students.

A concept map was introduced by Novak and Gowin [3] based on Ausubel's assimilation theory, which emphasized meaningful learning principles. Meaningful learning is defined as a process of linking new information to relevant concepts contained in a cognitive structure. Many researchers use the concept map to assess meaningful learning through students knowledge structure. But little effort has been made to extend the concept map in facilitating enhanced meaningful learning. Meaningful learning can be improved by connecting prior existing concept maps to new related information.

Extended concept mapping is an appealing and promoting approach to improve student's achievements in learning. The extended concept mapping structure is efficient in organizing design work and building a designer knowledge base [4] Extensible concept mapping at least provides two related activities that allow the structure of expansion efficiently 
organized and potentially improves problem-solving. Extending activities play an essential task in each phase of the knowledge integrating process through reviewing prior ideas and connections, eliciting missing ideas and relationships, and adding new concepts and relationships [5]. The extended mapping activity facilitates learners to expand the prior existing concept map and associate with further related information to realize enhanced meaningful learning.

A previous study [6] had developed an Extended ScratchBuild (ESB) concept map tool in facilitating meaningful learning enhancement. ESB allows the students to extend their prior concept map by adding new concepts, links, and propositions related to the material topic. ESB performance has also been investigated and stated a positive effect on students' learning outcomes. Nevertheless, there is no information concerning the achievement of concept maps components between original and additional parts. Clariana [7] believes that the number of concept map components defined by students illustrates the broad of information collected and elaborated by them.

The present study explored the effects of ESB in improving learners' knowledge structure. The performance of ESB was evaluated using achievements based on the amount of concept map components consisting of (1) number of concepts, (2) number of links, and (3) number of propositions. This study hypothesizes that the map expansion approach on ESB can improve learner's knowledge structure in quantity.

\section{EXTENDED SCRATCH-BUILD CONCEPT MAP}

Extended Scratch-Build (ESB) concept map is a graphical tool aimed to strengthen meaningful learning [6]. ESB is a computer-based and client/server concept mapping tool to support and improve learning services. Based on the system architecture that supports it, ESB tools can be implemented in various learning styles, including face-to-face or traditional learning, e-learning, and blended learning.

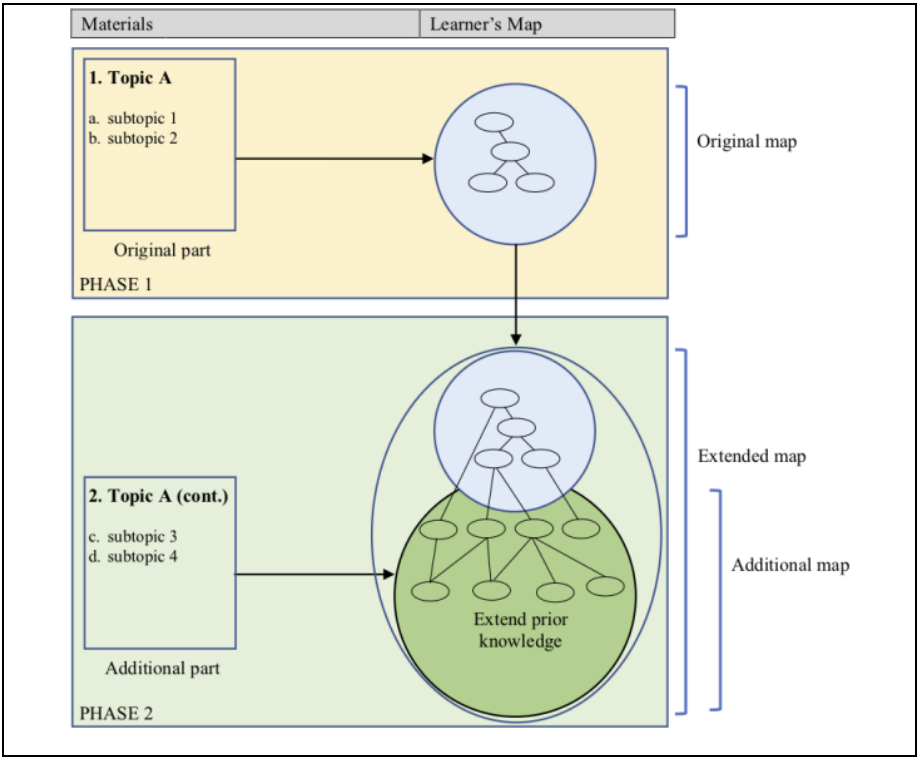

Fig. 1. The practical flow of ESB concept mapping
ESB inspired by open-ended concept mapping techniques that experienced to capture differences across students' knowledge structures. An open-ended method is a potential approach for reflecting and measuring students' knowledge structure. Still, it has a drawback in the achievement of understanding scores that tends to be less than closed-ended [8]. As explained in Fig. 1 [9], ESB attempts to overcome the shortcomings of the usual open-ended method by offering an expansion of a two-phase concept map: Phase 1 and Phase 2.

The expansion of the concept map facilitates learners to provide a well-organized structure and realize improved meaningful learning. Before applying ESB in practice, the teacher divided the material topic into two parts: original and additional parts. The original part is the material given to learners for the first phase of learning, while the additional part is the advanced material that would be provided in the second teaching. Original and additional parts are the same material in a particular subject.

In Phase 1, learners are asked to build a concept map in accordance with the first material or original map using an open-ended approach. Learners will get a blank canvas and are allowed to add concepts, links, and define propositions correspond to their understanding. This method is also known as construct a map from scratch.

Furthermore, in Phase 2, learners will be requested to expand their original concept map by adding new concepts, relationships, and propositions. In this situation, learners have the opportunity to review ideas and connections that have been made, reveal missing ideas and links, and add new concepts and relationships. Learners are also allowed to modify their previous concept maps if necessary. The activities in Phase 2 were yielding an extended map, which is a combination of the original map and an additional map.

The previous study [9] utilized the ESB approach in the Database 1 subject, which consists of fundamental concepts. The employment of concept maps in theoretical courses is considered appropriate and fulfills the conditions of meaningful learning [3]. The results reported that ESB had a positive effect on students' achievements, consist of understanding and map scores. The experimental results stated that the students' post-test scores after receiving learning with ESB support increased significantly compared to the pre-test. Additional map scores were also superior to the original map. Although the ESB performance has been confirmed, there is no information yet about the achievement of concept maps components.

\section{METHODOLOGY}

\section{A. Participants and Context of Study}

The present study involved 27 students from the Department of Electrical Engineering, State University of Malang, Indonesia. Participants are Informatics Engineering students who are familiar with the use of computers and the Internet in learning. All participants are novices in the experience of using concept mapping. 
This study was conducted in the Database 1 subject, and the lecturer was delivered the lecture material using Indonesian. The Relational Database topic was chosen, and based on the system design, the content was divided into two parts: original and additional contents. The original part material consists of 10 slides ( 283 words), and the second part material consists of 8 slides (237 words). A senior and experienced lecturer who had been teaching for 11 years was involved in this experiment.

\section{B. Experimental Procedure}

The ESB client application was run using a browser and accesses the server through an Internet network. Each student was given an account as a unique identity to access the extended mapping system. During concept mapping, the lecturer acts as a facilitator. Before the experiment was conducted, in a previous course meeting, participants had been given an introduction to concept maps and their use practices.

The experimental activities follow the flow, as shown in Fig. 2. The teacher begins by distributing printed material handouts to students. Furthermore, the teacher delivered the first material using a face-to-face approach for 25 minutes. In this situation, students were engaged in learning and actively participated as usual. Next, students were instructed to construct the concept map related to the first material for 15 minutes. During the concept maps creation, students were allowed to read their printed handouts that had been distributed beforehand. The final step in Phase 1 was uploading the original map creation results to the server.

In Phase 2, the lecturer continued giving the lecture regarding the additional part material. The second lecture was conducted with the same approach and time allocation, which was $25 \mathrm{~min}$. After that, students were asked to extend the original map by adding new concept maps components. The additional map is a map expansion results that are used to measure students' competence.

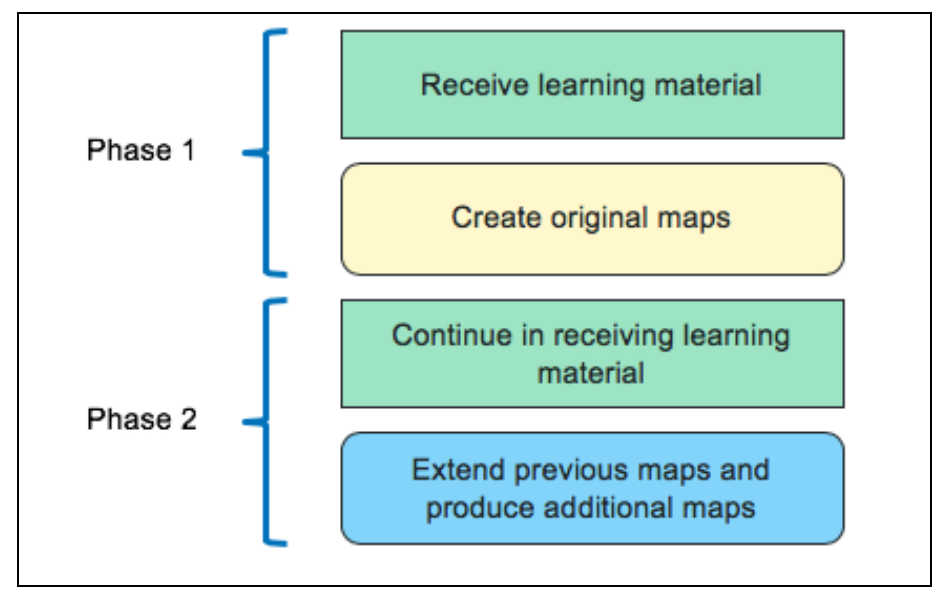

Fig. 2. The experimental activities

\section{Measurement}

The performance of ESB on students' knowledge structure was investigated using three concept map components measurement: (1) Number of concepts, (2) number of links, and (3) number of propositions. The number of concept map components can be obtained automatically using the system.

The number of propositions was identified through the number of links connected to two concepts and forms a valid relationship. Proposition evaluations were performed on all existing propositions made by each student, both scientifically correct and incorrect. The proposition is the smallest semantic unit in concept maps and represents the broadness of student knowledge.

\section{Data Analysis}

The dataset of concept map component was obtained from the original and additional maps separately. As explained in Fig. 1, the additional map illustrates the knowledge structure of expansion activity results. Thus, the increase in knowledge structure was analyzed through a comparison of the number of original maps and additional maps.

The normality distribution was examined to determine whether the data could be analyzed using a parametric test. The Shapiro-Wilk test was conducted on the paired difference scores, and it appears to be approximately normal. Thus, a parametric statistical analysis using a paired t-test was used to analyze the experimental results.

\section{RESULTS AND DISCUSSION}

\section{A. Analysis of Concept Map Components}

Concepts describe individual ideas. The number of concepts represents the breadth of a person's ideas in a particular domain of knowledge. Descriptive statistics of the number of concepts for original and additional maps are shown in Table I. Both maps show the same lowest score, which is 5. While for the highest score, the additional map is superior, which is 29 . The average achievement states that the expanded map is superior to the original map, 13.37 compared to 10.37 .

TABLE I. DESCRIPTIVE STATISTICS OF THE NUMBER OF CONCEPTS IN ORIGINAL AND ADDITIONAL MAPS

\begin{tabular}{lclllcc}
\hline \multicolumn{1}{c}{ Pair } & N & Min & Max & Median & Mean & SD \\
\hline Original map & 27 & 5 & 14 & 11.00 & 10.37 & 2.39 \\
Additional map & 27 & 5 & 29 & 13.00 & 13.37 & 5.75 \\
\hline
\end{tabular}

Based on data normality, the statistical analysis paired ttest was used to determine whether the number of concepts between the original and additional maps was a significant difference. The results showed a statistically significant difference between both concept maps $(\mathrm{t}=-2.601 ; \mathrm{p}=.016<$ $.05)$ with Pearson's $r$ was .452 , indicating a medium effect size.

The same quantity calculation was done for the links component that represents the relationships between ideas. Descriptive statistics of the number of links for both maps are shown in Table II. Achievement for this component was quite similar to the concepts component. The lowest links scores on the original and additional maps showed the same value, which was 6. For maximum scores, additional maps were 
consistent with high values, which was 30 , while the first map was 13. Similarly, on the average achievement of the links component, additional maps achieved the highest scores than the original map, 13.33 and 10.33 .

TABLE II. DESCRIPTIVE STATISTICS OF THE NUMBER OF LINKS IN ORIGINAL AND ADDITIONAL MAPS

\begin{tabular}{cclllcc}
\hline \multicolumn{1}{c}{ Pair } & N & Min & Max & Median & Mean & SD \\
\hline Original map & 27 & 6 & 13 & 10.00 & 10.33 & 1.69 \\
Additional map & 27 & 6 & 30 & 13.00 & 13.33 & 5.78 \\
\hline
\end{tabular}

The statistical analysis paired t-test was used to figuring out whether the number of links between the original and additional maps was a difference. Based on a significance level p-value threshold of 0.05 , there was a statistically significant difference between both concept maps $(\mathrm{t}=-2.582$; $\mathrm{p}=.015<.05)$ with Pearson's $\mathrm{r}$ was .454 , showing a medium effect size.

Descriptive statistics of the number of propositions in original and additional maps are shown in Table III. A proposition is the smallest unit and fundamental element in the concept map. The results denoted that the lowest value of map expansion was 4 , slightly superior to the original map, which was 3 . The achievement of the highest value in the additional map also showed significant results. As a result, the average number of propositions of the extended map was higher, which was 13.59 , compared to the original map, which was 8.85 .

TABLE III. DESCRIPTIVE STATISTICS OF THE NUMBER OF PROPOSITIONS IN ORIGINAL AND ADDITIONAL MAPS

\begin{tabular}{ccccccc}
\hline \multicolumn{1}{c}{ Pair } & N & Min & Max & Median & Mean & SD \\
\hline Original map & 27 & 3 & 13 & 9.00 & 8.85 & 2.80 \\
Additional map & 27 & 4 & 29 & 12.00 & 13.59 & 5.31 \\
\hline
\end{tabular}

Further statistical analysis was carried out to investigate differences in the achievement of the number of propositions on the original and additional maps. The results showed a statistically significant difference between both concept maps $(\mathrm{t}=-4.321 ; \mathrm{p}=.001<.05)$ with Pearson's $\mathrm{r}$ was .646, showing a large effect size. Cohen's Pearson's $r$ correlation value for both maps also indicated a large effect size $(r=.646)$.

\section{B. The Effects of ESB on Knowledge Structure Improvement}

The concepts are ideas or words which depict objects or events in the universe. The open-ended approach is recognized as particularly appropriate for expressing individual ideas and capturing differences among students [8]. Fig. 3 shows the results of achievements on the original and additional maps. In ESB concept mapping Phase 1, students had succeeded in extracting their ideas based on learning material. Furthermore, students were not only able to add new relevant ideas but also obtained more numbers of concepts in additional maps.

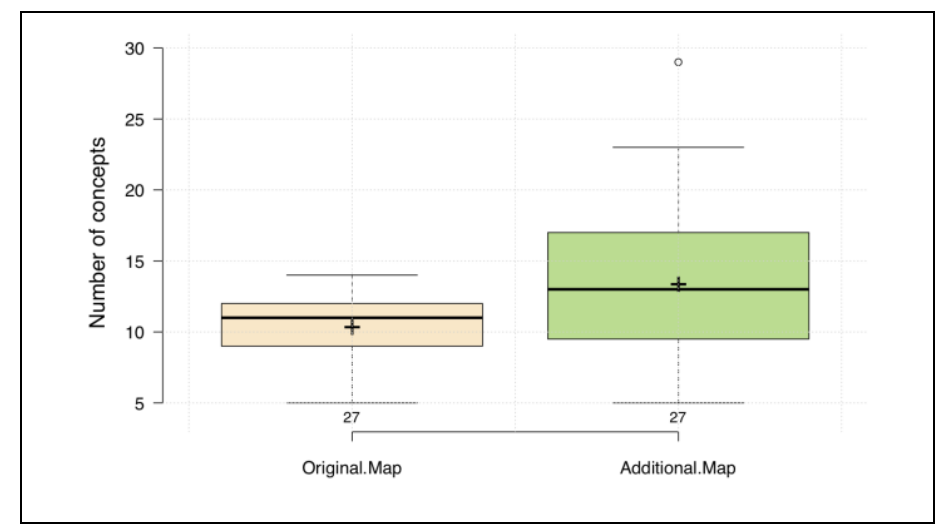

Fig. 3. Average achievement of concepts component in original and additional map

Phase 2 is a crucial stage that illustrates the main potential of ESB concept mapping. Although this phase also has the same time allocation as the first phase, it looks yield different achievements. The expansion phase provides an opportunity for learners to review and discover new ideas. This condition encourages them to produce broader expansion components. The existence of Phase 1 is a guideline for students to find relevant ideas and uncover new potential ideas.

The links or relationship components have a vital role in connecting two ideas and form a semantic unit. In Phase 1, students had succeeded in externalizing information and defining relevant links. The map expansion activities in Phase 2 encourage them to find more related links based on additional material, as shown in Fig. 4. It appears that students were able to reach a higher number of links on the additional map.

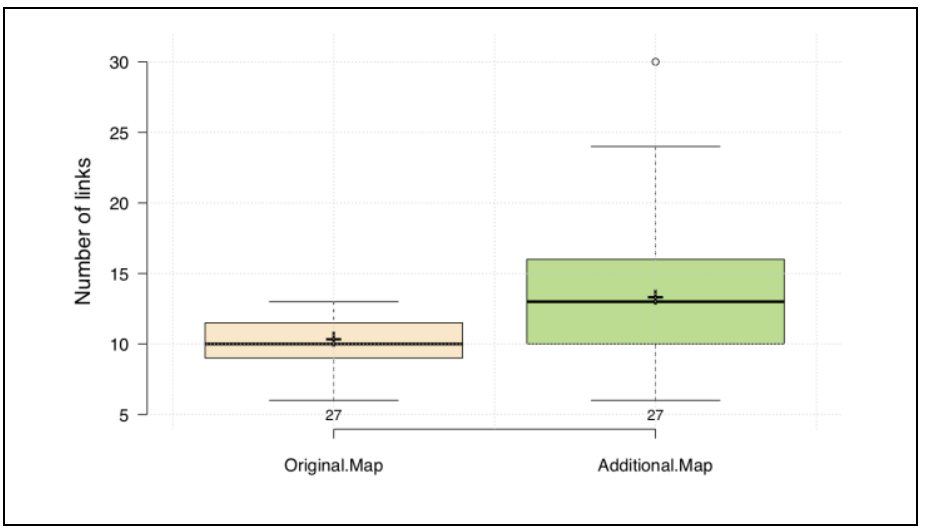

Fig. 4. Average results of links component in original and additional map

The open-ended approach is recognized as particularly appropriate for expressing personal knowledge and capturing differences among students [8]. Fig. 5 shows the results of achieving propositions on the original and additional maps. Phase 1 of the ESB concept mapping that utilizes the usual open-ended method confirms that students could form propositions according to their thinking. Furthermore, Phase 2, which offers map expansion, triggers students not only to be able to add new propositions regarding the material topic but also obtained more propositions. 


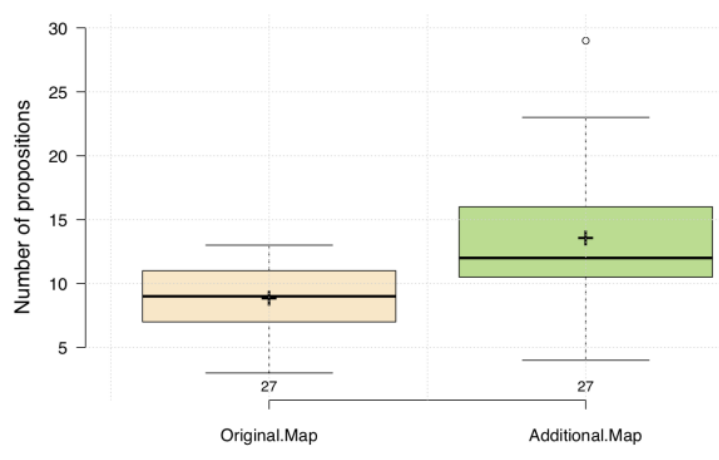

Fig. 5. Average achievement of propositions in original and additional map

Although Phase 2 provides the same time allocation as Phase 1, students were able to increase the number of propositions. The existence of propositions on previous maps stimulates them to connect previous schemes with new knowledge in a well-organized manner. Also, students could find new related propositions easily.

The statistical analysis reported that achieving additional maps outperformed original maps in terms of a number of concepts, links, and propositions. Although some researchers argue that the number of components does not always indicate a good concept map, it represents the broadness of individuals' knowledge structure [7], [10]. ESB concept mapping has proven that the expansion approach can improve learners' knowledge structure.

\section{CONCLUSION AND FUTURE WORKS}

This study explored the Extended Scratch-Build (ESB) concept mapping and investigated its effect on learners' knowledge structure. The main contribution of the current study was to observe the impact of extended mapping activities on students' knowledge structure achievements. The results of experiments on Database 1 subject, which consist of fundamental concepts, revealed that ESB concept mapping could improve the broadness of students' knowledge structure. The finding was evidenced by the achievement of concepts, links, and propositions components on the additional map that significantly increased compared to original maps.

Future works should involve the comparison group to investigate the performance of ESB in terms of knowledge structure achievements. Quality measurement is also needed to strengthen the findings of improvement in students' knowledge structure.

\section{Acknowledgments}

The first author would like to acknowledge the Islamic Development Bank (IsDB), the Universitas Negeri Malang (UM), Indonesia, and the Dept. of Information Engineering, Hiroshima University, Japan, for invaluable supports.

\section{References}

[1] T. Hirashima, "Reconstructional concept map: automatic Assessment and reciprocal reconstructIon," Int. J. Innov. Creat. Chang., vol. 5, no. 5, pp. 669-682, 2019.

[2] T. Hirashima, K. Yamasaki, H. Fukuda, and H. Funaoi, "Framework of kit-build concept map for automatic diagnosis and its preliminary use," Res. Pract. Technol. Enhanc. Learn., vol. 10, no. 1, p. 17, 2015.

[3] J. D. Novak and D. B. Gowin, Learning how to learn. New York: Cambridge University Press, 1984.

[4] D. Foley, F. Charron, and J. S. Plante, "Potential of the Cogex Software Platform to Replace Logbooks in Capstone Design Projects," Adv. Eng. Educ., vol. 6, no. 3, pp. 1-25, 2018.

[5] B. A. Schwendimann and M. C. Linn, "Comparing two forms of concept map critique activities to facilitate knowledge integration processes in evolution education," J. Res. Sci. Teach., vol. 53, no. 1, pp. 70-94, 2016.

[6] D. D. Prasetya, T. Widiyaningtyas, S. C. Putro, T. Hirashima, and Y. Hayashi, "Extended Scratch-Build Concept Map to Enhance Meaningful Learning," in 2019 International Conference on Electrical, Electronics and Information Engineering (ICEEIE), 2019, pp. 187-191.

[7] R. B. Clariana, T. Engelmann, and W. Yu, "Using centrality of concept maps as a measure of problem space states in computer-supported collaborative problem solving," Educ. Technol. Res. Dev., vol. 61, no. 3 , pp. 423-442, 2013.

[8] M. A. Ruiz-Primo, S. E. Schultz, M. Li, and R. J. Shavelson, "Comparison of the reliability and validity of scores from two concept-mapping techniques," J. Res. Sci. Teach. Off. J. Natl. Assoc. Res. Sci. Teach., vol. 38, no. 2, pp. 260-278, 2001.

[9] D. D. Prasetya, T. Hirashima, and Y. Hayashi, "Study on Extended Scratch-Build Concept Map to Enhance Students' Understanding and Promote Quality of Knowledge Structure," Int. J. Adv. Comput. Sci. Appl., vol. 11, no. 4, pp. 144-153, 2020.

[10] T. Stoddart, "Using concept maps to assess the science understanding and language production of English language learners," in Proc. of the Second Int. Conference on Concept Mapping, 2006. 